\title{
Aesthetic Reconstruction of Peno-Scrotal Lymphedema: Refinements of Previous Technique
}

\author{
ABD EL-RAHMAN M. ABD EL-AAL, M.D.; MINA ESTAWROW, M.D. and IKRAM SAFE, M.D. \\ The Department of Plastic and Maxillofacal Surgery, Faculty of Medicine, Ain Shams University, Cairo
}

\begin{abstract}
Background: Lymphedema is emotionally frustrating disease due to its progressive course and no definite curative methods of treatment, it was found that lymphedema results from local and/or systemic failure of the lymphatic transport system, and according to its cause and onset of lymphedema it is classified into primary and secondary lymphedema. Treatment methods of scrotal lymphedema and reconstruction were limited in the literature to lymphangioplasty or direct excision of the involved tissue and local tissue reconstruction. The current study present patients with established scrotal lymphedema and the way of surgical management in excision and reconstruction.
\end{abstract}

Patients and Methods: Ten male patients were involved in the study, their age was ranging between 25-60 years, three patients were primary lymphedema, seven patients were secondary lymphedema.

Results: Patients in the study were followed-up postoperatively for a minimum 6-month period, it was found that a clear improvement of the aspect of the external genitalia and subsequent improvement of ambulation, hygiene, and ability to void in the standing position occurred for all patients.

Conclusion: The procedure is effective giving good results with no major complications were recorded, the technique based on anatomical background that is why recurrence was not recorded.

Key Word: Scrotal - Lymphedema - Penile - Inguinal lymph nodes.

\section{INTRODUCTION}

The lymphatic system is responsible for transferring the interstitial fluid from different tissues and parts of the human system and returns it as lymph, which is rich in proteins and fats, back into the blood circulation [1]. Lymphatic channels in the periphery play an essential role to enhance the immune system as it transports leukocytes, regulate the immune response as well as the processing of both self-antigens and foreign antigens [2], that is why lymphatic mal function has been shown to predispose to bacterial infections and malignancies $[3,4]$.
Lymphedema can be defined as a progressive debilitating condition which have local and/or systemic failure of the lymphatic transport system, and it can be classified into primary and secondary lymphedema.

Primary lymphedema results from structurally and functionally abnormal lymphatic channels caused by obstruction, malformation, or hypoplasia. When primary lymphedema is present at birth or during infancy, it is known as congenital Milroy disease; however, the presentation of symptoms may occur later in life, such as in Meige disease (lymphedema praecox) (which manifests during adolescence) or lymphedema tarda (which usually becomes apparent after the age of 30 years).

On the other side, secondary lymphedema is caused by the destruction or obstruction of normally formed lymphatic channels. Although the most common cause of secondary lymphedema worldwide is filariasis, but secondary lymphedema can be a consequence of cancer treatment [5].

Lower limb lymphedema in general is an emotionally incapacitating condition, especially scrotal lymphedema and methods of reconstruction reported in the literature were limited to either lymphangioplasty or direct excision of the involved tissue with local tissue reconstruction [6].

Authors in the current study present patients with established scrotal lymphedema with their way of surgical management in excision and reconstruction.

\section{PATIENTS AND METHODS}

Current study is retrograde study which was done on patients with scrotal lymphedema presented to the authors in Ain Shams University Hospitals. 
Ten male patients were involved in the study, their age was ranging between 25-60 years, three patients were primary lymphedema, seven patients were secondary lymphedema with a history of filarial infestation early in their life, all patients were severe grade of peno-scrotal lymphedema detection of comorbid conditions as diabetes or hypertension was done as well as family history of similar conditions.

Examination was done for patient while standing primarily then in supine position, evaluation

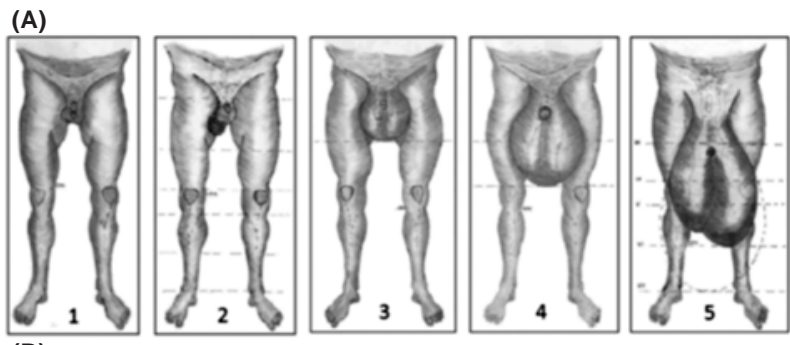

(B)

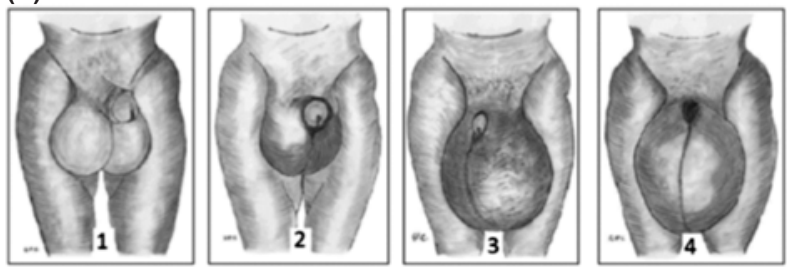

Photo (1): Diagram showing the grades and degrees of scrotal enlargement and buried penis.

(A) Scrotal enlargement stages 1-5 as described by Capuano et al., [7]: (1) Smaller than tennis ball, (2) Larger than tennis ball but not reaching mid femur, (3) Reaching mid femur but above patella, (4) Reaching patella but above tibial tuberosity, 5 below tibial tuberosity (dotted line stage reaching level of malleoli).

(B) Buried-penis grades 1-4: (1) Visible portion greater 2cm, (2) Visible portion less than $2 \mathrm{~cm}$, (3) Glans flush with skin, (4) Totally buried and irreducible.

\section{Surgical technique:}

Primarily, as a routine, hair removal in the pubic, perineal and inguinal region were performed then washing and cleaning of the whole scrotum and penis with betadine soap as well as the abdominal area and thighs down to the knees.

After that usual sterilization from the umbilicus down to the knee including the penis and scrotum.

Prophylactic broad-spectrum intravenous antibiotics were given. The original technique was published by the chief author 8 Photo (3) with aesthetic considerations in the current study, the procedure started by marking of the mid-inguinal point then draw a lateral curved incision from it (the incisions were marked at the transition between diseased and normal). involved the following size of scrotum and its stages (all patients were as graded as $5^{\text {th }}$ grade), lower limb edema (pitting or non-pitting), penis and penile skin with grading of buried penis in relation to symphysis pubis (patients were graded as $3^{\text {rd }}$ and $4^{\text {th }}$ ), lymph nodes and lymph vesicles as well as mons pubis area and its skin, and evaluation of skin of the mons whether is stretched or not as well as the length of suspensory ligament either increased or not. Patients were investigated by routine hematological tests and urine analysis.

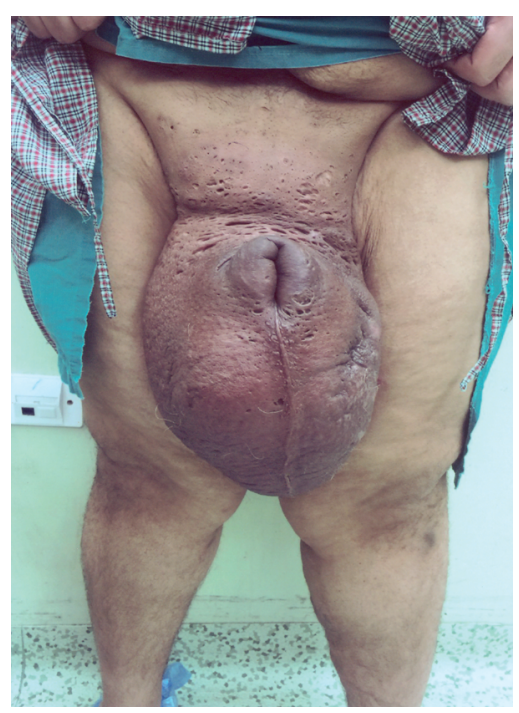

Photo (2): Pre-operative photo for a patient while standing.

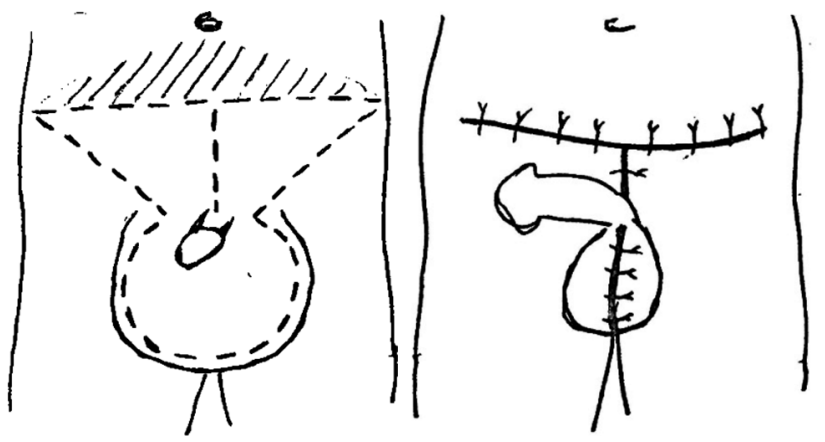

Photo (3): Diagrams of the original technique [8]: Showing design of the excision (Lt) \& pattern of closure (Rt).

The operation started by the lateral incisions through which, the spermatic cord is identified and delivered with associated dilated lymphatic trunks alongside the spermatic cord. These are separated and excised completely, the cord is better identified in the inguinal region. After doing the procedure on both sides, the elephantoid skin is removed till the scrotal base then the posterolateral skin is thinned in order to remove any pathological tissue. The penile skin was shaved from the coronal sulcus to the base (no way to preserve the penile skin), 
and according the extent of involvement of the mons area, the incision may continue from the root of the penis to continue the excision upward to meet the incision in the lower abdominal crease if the skin of the mons is severely distended or just make an opening in the mons in order to reposition the penis to its place. Testis was examined for hydrocele and excised if present.

After complete hemostasis, closure started posteriorly to obtain a scrotum with a size suitable to scrotal contents, closure was done in the midline simulating the scrotal raphe, the posterolateral skin was lifted up with three-point suture to meet the midline of the abdominal flap.

Closure was done in tension free manner with excision of excess skin, closure was done around

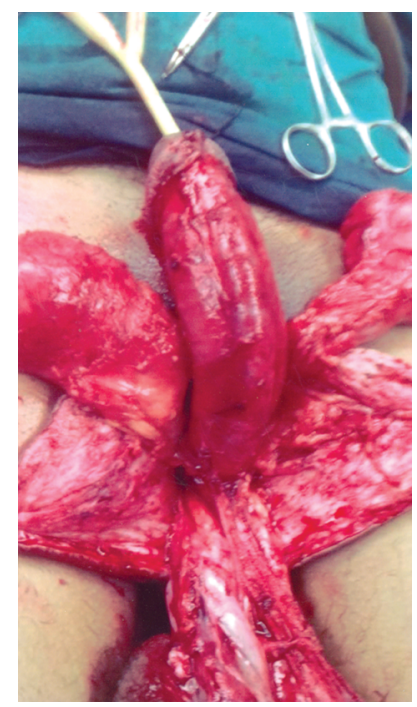

Photo (4): Intraoperative photo after surgical excision of the lymphedematous skin and dissection of the testes, shaft of the penis and spermatic cords.

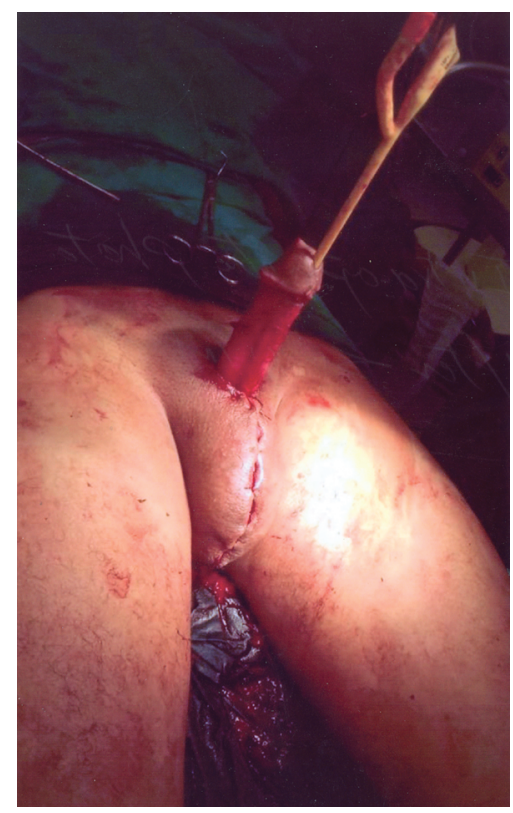

Photo (6): Intraoperative photo after surgical surgical closure. the penis, and a circular opening is done in the normal site of the root of the penis. Closure was completed in 2 layers, subcutaneous layer of $2 / 0$ vicryl, skin closed with $2 / 0$ silk sutures in a vertical mattress way.

The reconstructed scrotum was drained with 2 drains inserted through 2 different stabs, which was left in place till the output became lower than $50 \mathrm{ml}$.

Penis was covered with a split thickness skin graft harvested from the thigh with a tie-over dressing, the vertical suture better made in a zigzag fashion.

The patient remained with an indwelling urinary catheter for the same period. The scrotum was cleansed daily and kept with a scrotal suspensor.

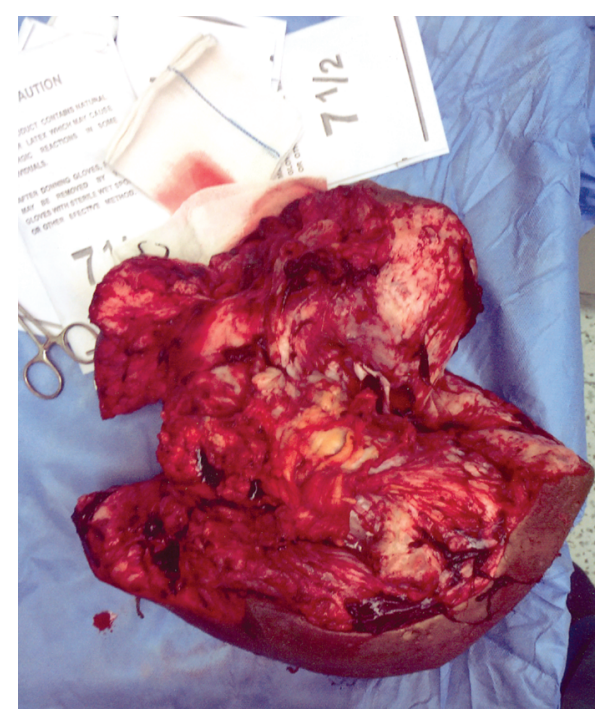

Photo (5): Intraoperative photo showing the excised lymphedematous skin.

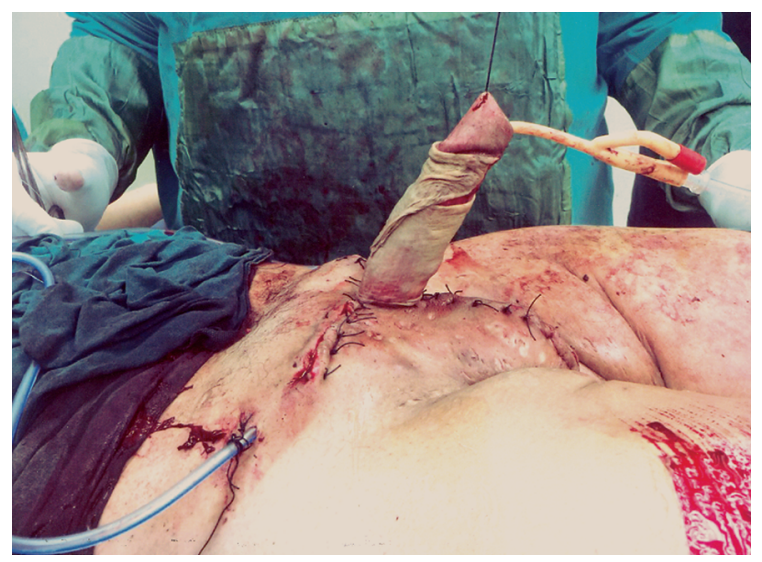

Photo (7): Intraoperative photo after application of STSG. 


\section{RESULTS}

Patients in the study were followed-up postoperatively for a minimum 6-month period, it was found that a clear improvement of the aspect of the external genitalia and subsequent improvement of ambulation, hygiene, and ability to void in the standing position occurred for all patients.

The improvement of sexual performance could not be directly assessed, although some patients stated that sexual intercourse became more effective as regards penetration and satisfaction for patient and partner.

Data regarding patients' ages, duration of the disease, size of scrotum and follow-up period are shown in (Table 1).

Patients had a favorable outcome all through their follow-up. There were no major complications recorded, although this surgical site is subject to contamination because of neighboring structures despite of that only 3 patients experienced little dehiscence in the vertical wound of the scrotum, but no surgical intervention was done and healed by 2 ry intention, otherwise no infections or necrosis was observed.

Table (1): Demographic data of the patients.

\begin{tabular}{lcccc}
\hline $\begin{array}{l}\text { Patient } \\
\text { no. }\end{array}$ & Age & $\begin{array}{c}\text { Duration of } \\
\text { disease (years) }\end{array}$ & $\begin{array}{c}\text { Size of } \\
\text { scrotum }\end{array}$ & Complications \\
\hline 1 & 25 & 4 & $14 \times 10$ & - \\
2 & 36 & 13 & $20 \times 15$ & Dehiscence \\
3 & 42 & 5 & $10 \times 30$ & Dehiscence \\
4 & 33 & 4 & $14 \times 11$ & - \\
5 & 29 & 10 & $15 \times 10$ & - \\
6 & 40 & 15 & $30 \times 12$ & Dehiscence \\
7 & 50 & 10 & $15 \times 25$ & - \\
8 & 44 & 12 & $13 \times 27$ & - \\
9 & 56 & 5 & $19 \times 24$ & - \\
10 & 60 & 13 & $15 \times 10$ & - \\
\hline
\end{tabular}

\section{DISCUSSION}

Scrotal lymphedema is a condition leading to the progressive enlargement of the scrotum and penis causing significant discomfort for patients [9].

With the progression of the lymphedema patient ambulation, sexual intercourse and voiding in the standing position with proper hygiene of the perineal region become difficult and even in some situations are impossible, as a sequalae for that malodor and recurrent episodes of skin infections as cellulitis and lymphangitis will be more frequent, that is why aggravation of the anatomopathological condition will occur as well as damage of the patient's quality of life and self-esteem [9].

Lymphedema of external genitalia either congenital or acquired. Non-congenital cases usually result from lymph node dissection, injury, or irradiation [6].

Different studies reported that inguinal area is drained by superficial and deep inguinal lymph channels, superficial channels are responsible for drainage of the scrotum and penile skin, diseased skin of the penis must be removed and replaced by skin graft as well as skin of the scrotum and replaced by flaps from nearby tissues, while the deep channels drain testes and penile body, that is why obstruction of the superficial channels will result in scrotal and penile lymphedema [10].

At present, it is generally agreed that the mainstay of therapy is surgical, as conservative measures usually of little value. Diuretics, elevation, and scrotal support have a limited effect on the regression of lymphedema except in mild cases and treatment of lymphangitis [6].

Many surgical innovative techniques have been evolved for scrotal and penile reconstruction. They were classified into physiologic and excisional procedures.

Physiologic operations were invented and designed to improve the lymph stasis condition by redirecting the static lymph fluid to the venous side. Huang et al., were the first surgeons who applied the concept of the lymphovenous shunt procedure for the treatment of male genital lymphedema, in which they performed anastomosis of the superficial lymphatic vessels to small veins in the subcutaneous tissue [11].

Mukenge et al., [12] chose deep lymphatic vessels along the spermatic cord and anastomosed them to the pampiniform venous plexus, which also runs beside the spermatic cord [11].

According to the lymphatic mapping of the inguinal region done by Sappy [13], there are two options for creating a lymphovenous shunt: Using the superficial lymphatic pathway or the deep lymphatic pathway, but the diameters of the superficial lymphatic vessels were smaller ( 0.2 to $0.5 \mathrm{~mm}$ ) compared with those of the deep lymphatic vessels ( 0.5 to $1 \mathrm{~mm}$ ); thus creating a lymphovenous shunt is technically more reliable using the deep lymphatic vessel. Another advantage of selecting the deep lymphatic vessels is that these vessels could be identified constantly beside the pampiniform 
veins [13], this technique has its limitations especially in cases of peno-scrotal lymphedema as patients usually need an effective rapid way of treatment [14].

On the contrary side, in chronic lymphedema there is a progressive fibrosis and obliteration of the collectors which is a characteristic feature of the disease, as well as the extent of skin resection determine the strategy used in lymphedema treatment. In excisional procedures strategies, they are either resection of the entire affected scrotal skin or minimization of the skin resection area for primary closure, various methods have been studied for resurfacing the excised raw surface. Skin grafting, it may have the advantage of reducing the recurrence of lymphedema and cellulitis, but thermal regulation of the testes is disturbed, that is why they addressed the other way by using local flaps from a normal thigh flap or a remnant of scrotal skin [13].

Delpech reported in his study in 1920, a successfully treated case of scrotal edema in which excision of lymphedematous tissue was followed by resurfacing of the penis and scrotum by local thigh flaps [6].

Sir Richard Henry Havelock Charles published a series of 140 consecutive patients which treated successfully of scrotal lymphedema. After that Sir Archibald McIndoe in 1950, performed the treatment of leg lymphedema with radical excision and skin grafting and attributed his work to Sir Havelock.

In 1985, Dandapat et al., [15] reported a case of elephantiasis of the scrotum and penis, in which excision of elephantoid skin and subcutaneous tissue was done through a vertical incision and suturing of the lateral scrotal neck skin in the midline for scrotal reconstruction.

Also, Martinez et al., displayed his work and his plan of management for three patients with lymphedema of the scrotum treated by radical excision of all lymphedematous scrotal tissue and reconstruction using posterolaterally based scrotal flaps [16], our technique is different in the position of the incisions as done in the junction between normal and pathologic skin with complete excision of the whole pathological tissues.

Huang [11] has tried a microlymphaticovenous procedure to treat elephantiasis of the scrotum and applied it clinically with good results.
Apesos and Anigian [17] presented a case of acquired genital elephantiasis in an elderly man after radical cystectomy and pelvic irradiation for transitional cell bladder cancer. Their plan of management involved the excision of the whole lymphedematous skin of the penis and scrotum, and reconstruction was done by the use of posterior scrotal flaps, superiorly based flap from the pubic area for testicular coverage, and split thickness skin graft for penile coverage, this technique has a disadvantage as they used a pathological tissues so recurrence rate is higher than in our technique.

Konety with his team [18] presented a case report of chronic hidradenitis of the perineum and scrotum with massive scrotal elephantiasis in which a wide resection of the scrotal mass and perineum was performed with reconstruction of the perineum and penis carried out using local skin flaps and split-thickness skin grafts.

Ndoye et al., [19] described his method scrotal reconstruction by anterior and posterior flap of scrotum after excision of the lymphatedematous mass.

Tammer et al., [20] reported a surgical technique for treating scrotal lymphedema by resection and neoscrotal reconstruction using ventral pedunculated scrotal skin flaps in cases of congenital hereditary of the Meige type.

All the previous studies are coincident with idea presented in the current study, as authors preferred the use of surgical excisional of the whole lymphedematous skin but they did not specify the point of starting of the excision and some of them used pathological tissues in reconstruction.

Here we started the excision from the junction with normal residual skin and complete removal of the penile skin depending on the anatomical background that all these structures are drained by superficial inguinal lymphatics and using local flaps from the remnant scrotal skin as this part is drained by deep lymphatic channels with skin grafting of penile shaft to avoid recurrence of lymphedema in this area and putting in consideration the aesthetic point of view of the overall groin area with reconstruction of the mons and lower abdominal area by either resection of the skin if severely involved or using it to reposition the penis by opening through this skin area, resulting in a normally aesthetically pleasing peno-scrotal area as well as mons pubis area.

The procedure took about 2-3 hours duration under general anesthesia for ten patients who 
involved in the study, all these patients experienced unremarkable follow-up with very good improvement of their lives and very good psychological improvement, they passed without major complications, except for 3 patients who suffered from little dehiscence in the vertical line. During the follow up period there was no evidence of recurrence in all patients.

\section{Conclusion:}

In conclusion, the current technique scientifically based on anatomical knowledge so the rate of recurrence is low, also the technique is easy, reliable without major complications.

\section{REFERENCES}

1- D'Alessandro A., Clement C.C. and Santambrogio L.: Lymph formation, composition and circulation: A proteomics perspective. Hansen KC, Int. Immunol., 27: 21927, 2015.

2- Liao S. and Von Der Weid P.Y.: Lymphatic system: An active pathway for immune protection. Semin. Cell Dev. Biol., 38: 83-9, 2015.

3- Ruocco V., Schwartz R.A. and Ruocco E.: Lymphedema: An immunologically vulnerable site for development of neoplasms. J. Am. Acad. Dermatol., 47: 124-7, 2002.

4- Rockson S.G.: The lymphatics and the inflammatory response: Lessons learned from human lymphedema. Lymphat. Res. Biol., 11: 117-20, 2013.

5- Current Concepts in the Surgical Management of Lymphedema.

6- Pramod Kumar M.S., M.Ch., D.N.B. and Gurusamipalayam Periyasamy Navaneethan, M.B.B.S. Resection of Scrotal Lymphedema.

7- Capuano G.P. and Capuano C.: Surgical management of morbidity due to lymphatic filariasis: The usefulness of a standardized international clinical classification of hydroceles. Trop. Biomed., 29: 24-38, 2012.

8- Ikram Safe, M.D.: Peno-scrotal lymphoedema; one-stage reconstruction. Egyptian Journal of Plastic \& Reconstructive Surgery. Jan., 21-8, 1993.

9- Farina R. and Farina G.: Elefantíase peno-escrotal (osqueofaloplastia). Rev. Bra. Cir., 85: 20512, 1995.

10- Modolin M., Mitre A.I., Da Silva J.C.F., Cintra W., Quagliano A.P., Arap S. and Ferreira M.C.: Surgical treatment of lymphedema of the penis and scrotum. Clinics, 61 (4): 289-94, 2006.

11- Huang G.K., Hu R.Q., Liu Z.Z. and Pan G.P.: Microlymphaticovenous anastomosis for treating scrotal elephantiasis. Microsurgery, 6: 36-9, 1985.

12- Mukenge S.M., Pulitanó C., Colombo R., Negrini D. and Ferla G.: Secondary scrotal lymphedema: A novel microsurgical approach. Microsurgery, 27: 655-6, 2007.

13- Sappey M.P.C.: Anatomie, Physiologie, Pathologie des vaisseaux lymphatiques. Paris: Adrien Delahaye, 1874.

14- Adil Kallat, Ahmed Ibrahimi, Hani Abousaleh, Hachem El-Sayegh, Ali Iken, Lounis Benslimane and Yassine Nouini: Scrotal Elephantiasis: About One Case and Literature Review. International Journal of Current Innovation Research, Vol. 3, Issue 04, pp 668-70, April 2017.

15- Dandapat M.C., Mohapatro S.K. and Patro S.K.: Elephantiasis of the penis and scrotum: A review of 350 cases. Am. J. Surg., 149: 686, 1985.

16- Martinez R.E., Couchell S.H., Raffel B. and Swartz W.M. Primary lymphedema of the scrotum: Surgical treatment and reconstruction. Ann. Plast. Surg., 21: 354, 1988.

17- Apesos J. and Anigian G.: Reconstruction of penile and scrotal lymphedema. Ann. Plast. Surg., 27: 570, 1991

18- Konety B.R., Cooper T., Flood H.D. and Futrell J.W.: Scrotal elephantiasis associated with hidradenitis suppurativa. Plast. Reconstr. Surg., 97: 1243, 1996.

19- Ndoye A., Sylla C., Ba M., Gueye S.M. and Diagne B.A.: Point of technique: Management of penile and scrotal elephantiasis. B. J. U. Int., 84: 362, 1999.

20- Tammer M.E., Plogmeier K. and Schneider W.: Surgical therapy of scrotal edema in elephantiasis congenita hereditaria (Meige type). Urologe A., 41: 493, 2002. 